\title{
Left Pulmonary Artery
}

National Cancer Institute

\section{Source}

National Cancer Institute. Left Pulmonary Artery. NCI Thesaurus. Code C32971.

A blood vessel arising from the bifurcation of the pulmonary artery that carries

deoxygenated blood to the left lung. 